\title{
Multivariate Profiles of Selected Versus non-Selected Elite Youth Brazilian Soccer Players
}

\author{
by \\ Rodrigo Aquino ${ }^{1,2}$, Isabella S. Alves ${ }^{3}$, Maickel B. Padilha ${ }^{1}$, Filipe Casanova ${ }^{1}$, \\ Enrico F. Puggina2,3, José Maia ${ }^{1}$
}

\begin{abstract}
This study determined whether a multivariate profile more effectively discriminated selected than non-selected elite youth Brazilian soccer players. This examination was carried out on 66 youth soccer players (selected, $n=28$, mean age $16.3 \pm 0.1$; non-selected, $n=38$, mean age $16.7 \pm 0.4$ ) using objective instruments. Multivariate profiles were assessed through anthropometric characteristics, biological maturation, tactical-technical skills, and motor performance. The Student's $t$-test identified that selected players exhibited significantly higher values for height $(t=2.331, p=0.02)$, lean body mass $(t=2.441, p=0.01)$, and maturity offset $(t=4.559, p<0.001)$, as well as performed better in declarative tactical knowledge $(t=10.484, p<0.001)$, shooting $(t=2.188, p=0.03)$, dribbling $(t=5.914, p<0.001)$, speed $-30 \mathrm{~m}$ $(t=8.304, p<0.001)$, countermovement jump $(t=2.718, p=0.008)$, and peak power tests $(t=2.454, p=0.01)$. Forward stepwise discriminant function analysis showed that declarative tactical knowledge, running speed $-30 \mathrm{~m}$, maturity offset, dribbling, height, and peak power correctly classified $97 \%$ of the selected players. These findings may have implications for a highly efficient selection process with objective measures of youth players in soccer clubs.
\end{abstract}

Key words: sport selection, youth athletes, performance level, tactical analysis, skill acquisition.

\section{Introduction}

Adequate selection and placement processes of youth soccer players have been systematically linked to team success throughout the competitive seasons (Huijgen et al., 2014). Additionally, the opportunities of soccer players to reach professional levels are reduced for nonselected players, which reveals the importance of decision-making (Huijgen et al., 2014). Tactical, technical and physical attributes displayed as a multivariate profile can help in the discrimination between elite and non-elite, and selected and nonselected youth players and assist decision makers in predicting their future success (Gissis et al., 2006; Huijgen et al., 2014; Meylan et al., 2010). Success of youth soccer players is also dependent on a number of external factors namely practice opportunities, appropriate coaching across training seasons, and not receiving compromising injuries, as well as social, cultural and personal aspects (Reilly et al., 2000).

Usually, soccer scouts observe young players during competitions and select the best (Huijgen et al., 2014). However, little is known about the criteria with which these decisions are made. This decision-making process can be highly subjective and distinguishing between current ability and future potential is rather a difficult task, particularly during periods of rapid physical growth and development (Archer et al., 2016; Unnithan et al., 2012; Vaeyens et al., 2008). However, this process can be reinforced by objective measures, namely the use of an array of

\footnotetext{
1 - CIFI2D, Faculty of Sport, University of Porto, Porto, Portugal.

2 - Post-graduate Program in Rehabilitation and Functional Performance, Medicine School of Ribeirao Preto, University of São

Paulo, Ribeirao Preto, Brazil.

3 - School of Physical Education and Sport, University of São Paulo, Brazil.
} 
tests to help assess the multivariate profiles of the players. Since it is unknown how much solid experience scouts and soccer coaches have when making clinical decisions during the selection process in soccer clubs with low economic resources, the use of objective testing could be of great help (Huijgen et al., 2014).

A precise map of youth soccer player performance is somewhat difficult to obtain given the multifaceted combination of characteristics that affect what happens during the match (Carling et al., 2009). For example, physical performance has to be closely linked to technical skills, tactical actions, and strategic decisions. Therefore, we contend that objective data from an adequate battery of tests may help coaches in differentiating distinct levels of performance, i.e., between selected and non-selected soccer players, which will significantly reduce the time and costs of decision making. Recently, Honer et al. (2015) showed that physical tests together with soccerspecific technical skills (e.g. speed, dribbling, shooting) help in objectively differentiating between players of different performance levels. Furthermore, Huijgen et al. (2014) investigated sets of traits that distinguished between the Netherlands' selected and non-selected youth players and found that $69 \%$ of selected players were correctly classified in four domains (physical, technical, tactical and psychological). Similarly, Figueiredo et al. (2009) found that physiological, technical and psychological traits of young soccer players differed between drop-out players and those who remained at the club or were selected by elite clubs.

However, to the best of our knowledge, no previous studies have considered the joint effects of explosive strength, repeated sprint ability and maximal oxygen uptake (VO2max) together with game knowledge and soccerspecific technical skills. Therefore, we aimed to determine whether multivariate profiles (anthropometric characteristics, biological maturation, tactical skills, technical skills and motor performance) more effectively discriminated between selected than non-selected elite youth Brazilian soccer players.

\section{Methods}

\section{Participants}

Sixty-six soccer players aged $16.18 \pm 0.63$ years (selected, $\mathrm{n}=28$; non-selected, $\mathrm{n}=38$ ) participated in this study. Regarding their experience level, $92.85 \%$ (selected) and 10.52\% (non-selected) had participated in state and/or national championships before the start of the study. All players had been enrolled in a highlevel soccer club in São Paulo State (SP), Brazil, for at least one consecutive year. This club was among the best 37 clubs in the country and had the highest rate of soccer academies.

The study was approved by the Research Ethics Committee of the Faculty of Medicine at Ribeirao Preto (protocol 710.998/2014) and written assent was given by all players as well as consent by their parents or legal guardians.

\section{Procedures}

Early in the 2015 competitive season, 28 players were allowed to continue in the team (selected), while 38 players were informed of leaving this group (non-selected). This decision was made by club scouts and coaches $(\sim 15$ years of experience in the job). In this competitive season, $\sim 30$ weeks, the team (U-17 - composed solely of selected players) was ranked among the top four of the championship. At the time of this research, the championship of the U-17 category had a total of 66 teams.

Prior to the study, players trained four times per week ( $100 \mathrm{~min}$ per session) and played regional competitive games once per week (every Saturday). The majority of training sessions consisted of tactical-technical training ( $70 \%$ of the total volume). The other $30 \%$ were dedicated to exercises aimed at strength and speed development. The assessment protocol for this study was developed prior to the selection process, and all measurements were conducted over two weeks.

\section{Measures}

Anthropometry

Stretched height $(\mathrm{cm})$ and seating height $(\mathrm{cm})$ were measured with the head positioned in the Frankfurt plane using a fixed wall stadiometer (Sanny Professional-ES2020, Brazil) with precision of $0.1 \mathrm{~cm}$. Body mass $(\mathrm{kg})$ was measured with subjects wearing light clothing on a digital scale (Filizola, PL 200, Brazil) with $100 \mathrm{~g}$ precision. Triceps, subscapular, chest, midaxillary, suprailiac, abdominal and mid-thigh skinfolds were measured according to available protocols (Lohman, 1986), and used to calculate \% body fat 
and lean body mass using Jackson et al. (1978), and Siri (1961) equations, respectively. All measurements were made with a Harpenden caliper (British Indicators Ltd., UK) with $0.2 \mathrm{~mm}$ accuracy. Each skinfold was measured three times, and the median value of the three measures was used for further analysis.

Biological maturation

Biological maturation was assessed by the maturity offset procedure proposed by Mirwald et al. (2002), which estimates the age of occurrence of peak height velocity (PHV). The maturity offset estimates the distance each subject is from PHV, using chronological age and the value is expressed in decimal years. A positive (+) maturity offset represents the number of years the participant is beyond PHV, whereas a negative (-) maturity offset represents the number of years the subject is before PHV.

Tactical skill

An adapted version proposed by Giacomini et al. (2011) of declarative tactical knowledge was used in this study to estimate players' game knowledge. This methodology uses eight offensive soccer scenarios (11 vs. 11) to assess right or wrong answers in each action. Each scenario is presented and frozen for two seconds before the player decides "what to do". After the scenario is presented, four photographs pop up on the screen, ranked from 1 to 4 , with four different possible solutions. The player is required to select the best answer with no time limit. Scores for each answer are as follows: best solution (100\% accuracy), 1 point; second best solution (75\% accuracy), 0.75 point; third best solution (50\% accuracy), 0.5 point; worst solution $(25 \%$ accuracy), 0.25 point. The final score is the sum of the responses to all scenarios (minimum 2 and maximum 8 points).

\section{Technical skills}

Soccer-specific technical skills were assessed using three tests (shooting, ball control, and dribbling) proposed by the Portuguese Soccer Federation, which had been used in previous studies (Coelho-e-Silva et al., 2010; Matta et al., 2014). The shooting test consisted of players taking five shots at a goalpost $2 \mathrm{~m}$ in height and 3 $\mathrm{m}$ in width from a distance of $9 \mathrm{~m}$. The goalpost was divided into six spaces by three elastic bands (two placed vertically and two placed horizontally). Scores were as follows: 1 point (bottom central space), two points (upper central space), 3 points (side rectangles), and 5 points (upper corners, right and left). The final score was obtained by adding the individual scores of all five shots. In the ball control test, the player was required to keep the ball in the air without using their arms or hands in a space of $9 \times 9 \mathrm{~m}$. The final score was the number of ball touches. In the dribbling test, players were instructed to slalom dribble the ball around nine cones positioned in a straight line and separated by a distance of $2 \mathrm{~m}$. Participants were oriented to progress from the starting line to the final cone and return. The aim was to complete the course in the shortest time possible, and the time was recorded using Fspeed photocells (FEsystems ${ }^{\circledR}$, Brazil). Two attempts were allowed in the ball control and dribbling tests and the best performance was considered for further analysis.

Motor performance

Running speed was assessed using a speed test $-30 \mathrm{~m}$ (Little and Williams, 2005), in which players ran $30 \mathrm{~m}$ at full speed. The time was recorded using Fspeed photocells (FEsystems ${ }^{\circledast}$, Brazil). Jumping height was measured with the vertical jump (countermovement jump - CMJ) using the ergojump protocol (Bosco et al., 1995). This test consisted of performing a jump with a flexion and extension movement of the lower limbs, without movement of the upper limbs, using the ergojump platform (Cefise ${ }^{\circledR}$, Brazil). Two attempts were allowed for the speed $-30 \mathrm{~m}$ and CMJ and the best was retained. The Running Anaerobic Sprint Test (RAST) was used to assess players' repeated sprint ability according to Zacharogiannis et al. (2004). The players performed six sprints of $35 \mathrm{~m}$ at full speed with $10 \mathrm{~s}$ of passive recovery between each run, and the following variables were recorded: average power, peak power, minimum power and fatigue index. The time for each sprint was recorded using Fspeed photocells (FEsystems ${ }^{\circledR}, \quad$ Brazil). Aerobic performance $\left(V_{2}{ }_{2 m a x}\right)$ was measured by the Yo-Yo Intermittent Recovery Test - level 1 (Bangsbo et al., 2008). This test consisted of $2 \times 20 \mathrm{~m}$ shuttle runs at increasing speeds, controlled by audio signals. Between each running bout, players were allowed five seconds of active recovery.

Statistical Analysis

Basic results are presented as mean \pm 
standard deviation (sd), minimum (Min) and maximum (Max). Normality and homogeneity of variances were checked, and no violations were noticed. Hotteling's $\mathrm{T}^{2}$ was used to test for differences between selected and non-selected players' mean vectors of all variables. As a followup, Student's t-tests were applied to identify individual variables that significantly distinguished between the groups. Using only variables that were statistically significant, a forward stepwise discriminant function analysis (DF) was employed to identify the smallest set of variables that maximized differences between the groups. Finally, results from the classification matrix revealed how precise the smallest variable set obtained from the DF was in recovering the original grouping of all subjects. All analyses were performed in SYSTAT 13, and the level of significance was set at $5 \%$.

\section{Results}

Table 1 presents the descriptive statistics. In general, selected players were taller, had more lean body mass, and performed better in technical, tactical, and motor tests than nonselected players.

The multivariate test of differences between selected versus non-selected players was statistically significant, Hotteling $\mathrm{T}^{2}=326.45, p<$ 0.001. The follow-up Student's t-tests identified that in nine out of the 15 variables, the selected players exhibited significantly higher values for height $(\mathrm{t}=2.331, p=0.02)$, lean body mass $(\mathrm{t}=$ $2.441, p=0.01)$, and maturity offset $(t=4.559, p<$ $0.001)$, and performed better in tests of declarative tactical knowledge $(\mathrm{t}=10.484, p<$ $0.001)$, shooting $(t=2.188, p=0.03)$, dribbling $(t=$ 5.914, $p<0.001)$, speed $-30 \mathrm{~m}(\mathrm{t}=8.304, p<$ $0.001), \mathrm{CMJ}(\mathrm{t}=2.718, p=0.008)$, and peak power $(\mathrm{t}=2.454, p=0.01)$.

\begin{tabular}{|c|c|c|c|c|}
\hline \multicolumn{5}{|c|}{$\begin{array}{l}\text { Table } \mathbf{1} \\
\text { maximum [Max]) } \\
\text { ll, technical skills } \\
\text { youth soccer players. }\end{array}$} \\
\hline & $\begin{array}{c}\text { Selected } \\
(\mathrm{n}=28) \\
\mathrm{M} \pm \mathrm{sd}\end{array}$ & Min-Max & $\begin{array}{c}\text { Non-selected } \\
(\mathrm{n}=38) \\
\mathrm{M} \pm \mathrm{sd}\end{array}$ & Min-Max \\
\hline \multicolumn{5}{|l|}{ Anthropometry } \\
\hline 1. Body height $(\mathrm{cm})$ & $176.79 \pm 5.02$ & 167-187 & $173.87 \pm 5.025$ & $163-184$ \\
\hline 2. $\%$ body fat & $13.09 \pm 3.10$ & $6.41-21.70$ & $11.92 \pm 3.81$ & $5.62-22.14$ \\
\hline 3. Lean body mass (kg) & $30.92 \pm 2.06$ & $\begin{array}{l}26.02- \\
34.17\end{array}$ & $29.86 \pm 1.22$ & $\begin{array}{c}26.94- \\
31.89\end{array}$ \\
\hline \multicolumn{5}{|l|}{ Biological maturation } \\
\hline 4. Maturity offset (years) & $1.90 \pm 0.59$ & $0.97-2.82$ & $1.31 \pm 0.40$ & $0.56-2.43$ \\
\hline \multicolumn{5}{|l|}{ Tactical Skill } \\
\hline $\begin{array}{l}\text { 5. Declarative knowledge } \\
\text { tactical (pts) }\end{array}$ & $6.44 \pm 0.23$ & $5.75-6.75$ & $5.90 \pm 0.17$ & $5.50-6.25$ \\
\hline \multicolumn{5}{|l|}{ Technical Skills } \\
\hline 6. Shooting (pts) & $9.53 \pm 3.21$ & $4-14$ & $7.86 \pm 2.94$ & $2-12$ \\
\hline 7. Ball control (touches) & $104.35 \pm 54.23$ & $21-255$ & $106.18 \pm 57.21$ & $19-201$ \\
\hline 8. Dribbling (s) & $11.50 \pm 0.86$ & $\begin{array}{l}10.22- \\
13.54\end{array}$ & $12.85 \pm 0.94$ & $\begin{array}{l}11.21- \\
14.53\end{array}$ \\
\hline \multicolumn{5}{|l|}{ Motor Performance } \\
\hline 9. Cyclical speed-30m (s) & $4.12 \pm 0.12$ & $4.00-4.54$ & $4.53 \pm 0.26$ & 4.01-5.10 \\
\hline $\begin{array}{l}\text { 10. Countermovement jump } \\
(\mathrm{cm})\end{array}$ & $35.49 \pm 1.81$ & $\begin{array}{l}31.34- \\
37.99\end{array}$ & $34.14 \pm 2.13$ & $\begin{array}{l}30.01- \\
38.78\end{array}$ \\
\hline 11. Average power $\left(\mathrm{W} \cdot \mathrm{kg}^{-1}\right)$ & $6.87 \pm 0.56$ & $5.56-8.13$ & $6.58 \pm 0.87$ & $4.61-8.23$ \\
\hline 12. Peak power $\left(\mathrm{W} \cdot \mathrm{kg}^{-1}\right)$ & $8.54 \pm 0.71$ & $6.72-9.71$ & $8.00 \pm 0.97$ & $5.59-9.49$ \\
\hline 13. Minimum power $\left(\mathrm{W} \cdot \mathrm{kg}^{-1}\right)$ & $5.07 \pm 0.61$ & $3.17-5.98$ & $4.81 \pm 0.69$ & $3.57-6.07$ \\
\hline 14. Index of fatigue $(\%)$ & $6.92 \pm 2.09$ & $3.04-10.76$ & $6.41 \pm 2.27$ & $2.88-11.58$ \\
\hline 15. $V \mathrm{O}_{2 \max }\left(\mathrm{ml} \cdot \mathrm{kg}^{-1} \cdot \mathrm{min}^{-1}\right)$ & $52.82 \pm 3.18$ & $\begin{array}{c}47.40- \\
58.98\end{array}$ & $51.87 \pm 2.94$ & $\begin{array}{c}46.01- \\
57.87\end{array}$ \\
\hline
\end{tabular}




\begin{tabular}{|c|c|c|c|c|}
\hline \multicolumn{5}{|c|}{$\begin{array}{r}\text { Table } 2 \\
\text { Summary of stepping summary in forward stepwise discriminant analysis. }\end{array}$} \\
\hline Step & Entered & Wilks's Lambda & Approx. F-Ratio & $p$ \\
\hline 1 & $\begin{array}{l}\text { Declarative tactical knowledge } \\
\text { (pts) }\end{array}$ & 0.368 & 109.919 & $<0.001$ \\
\hline 2 & Running speed $-30 \mathrm{~m}(\mathrm{~s})$ & 0.285 & 79.186 & $<0.001$ \\
\hline 3 & Maturity offset (years) & 0.222 & 72.324 & $<0.001$ \\
\hline 4 & Dribbling (s) & 0.209 & 57.576 & $<0.001$ \\
\hline 5 & Height $(\mathrm{cm})$ & 0.196 & 49.181 & $<0.001$ \\
\hline 6 & Peak power $\left(\mathrm{W} \cdot \mathrm{kg}^{-1}\right)$ & 0.187 & 42.632 & $<0.001$ \\
\hline
\end{tabular}

\begin{tabular}{|cccc|}
\hline & & \\
& & & Table 3 \\
Classification matrix of selected and non-selected & elite Brazilian youth soccer players. \\
\hline & Selected & Non-selected & \% correct \\
\hline Selected & 26 & 2 & 93 \\
Non-selected & 0 & 38 & 97 \\
\hline Total & 40 & 26 & \\
\hline
\end{tabular}

Table 2 reports the main results of the forward stepwise DF and shows the smallest set of the previous nine variables that best discriminated between the groups. The order of importance is as follows: declarative tactical knowledge, running speed - $30 \mathrm{~m}$, maturity offset, dribbling, height, and peak power.

From the classification matrix results (Table 3), all non-selected players were correctly classified in their original group, while in the selected players only two were misclassified. The percentage of correct classifications was 97\%, which was confirmed by the Jackknife leave-oneout method. This method is a cross-validation technique which excludes one observation, formulates a discriminant function using the remaining data, and uses that function to classify the excluded observation. The advantage is that the classification is totally independent of the excluded sample.

\section{Discussion}

This study investigated the power of multivariate profiles to discriminate between selected versus non-selected elite Brazilian youth soccer players. The combined effects of declarative tactical knowledge, running speed $30 \mathrm{~m}$, maturity offset, dribbling, height, and peak power accounted for $97 \%$ of correct classifications of the selected players. Furthermore, the effectiveness of these multiple objective measures can support and validate the decision-making 
processes. Club decisions regarding the maintenance or drop-out of youth soccer players are important pronouncements for their future career, i.e., the likelihood a player has to reach the professional level given the potential of their multivariate profiles (Huijgen et al., 2014). The more objective the information is, the higher the chances of a club decision-maker finding the most appropriate answer in their actuarial prediction (Vaeyens et al., 2006). The critical issue of clinical versus actuarial or statistical prediction has been widely discussed in several domains where decision making under uncertainty is mandatory (Dawes et al., 1989). Club scouts and coaches (i.e., the clinicians) who selected the players in our study wer expert raters with $\sim 15$ years of experience in the job, and this most likely contributed to the high precision of their classification (97\%). Yet, based on players' multivariate profiles, two of them were incorrectly classified as belonging to the nonselected group. This situation most probably arose because they were below the others in biological maturation.

The selection process has long been of great interest to sports coaches for various reasons, namely the determination of appropriate developmental processes to achieve success in response to training and competition (Lago-Penas et al., 2014; Reilly et al., 2000). Besides tactical and technical skills, players' physical growth, biological maturation and motor performance are essential to discriminate between selected and non-selected youth soccer players. In our study, height and lean body mass results were unexpected given that previous reports had shown them not to be useful (Huijgen et al., 2014; Lago-Penas et al., 2014; Reilly et al., 2000). Malina et al. (2004) showed that biological maturation significantly contributed to establishing mean differences in youth soccer players' physical growth and motor performance, i.e., favored those advanced in puberty. Furthermore, it is well acknowledged that, in non-athletic young people, those ahead in biological maturation are taller, weigh more and have higher lean body mass (Malina et al., 2004), and the same trend occurs in youth athletes (Blanksby et al., 1994).

Knowledge of the game is a highly regarded asset in youth soccer players, and in the present study we analyzed their declarative tactical knowledge. This variable proved to be the most important one in the discriminant analysis. Similarly, Huijgen et al. (2014) reported that a complex set of technical (peak dribbling), tactical (positioning and deciding) and physiological (peak sprinting) characteristics correctly classified $69 \%$ of the selected players, and the technical aspect was the most important.

It has been shown that, on average, technical skill levels differ significantly between selected and non-selected, as well as elite and non-elite soccer players (Honer et al., 2015; Huijgen et al., 2009, 2010, 2014; Rebelo et al., 2013). In our study, shooting and dribbling tests favored selected players, and this can be partly explained by the fact that they were ahead in biological maturation. Likewise, Malina et al. (2005) estimated the contribution of maturity status to variation in sport-specific skills of adolescent soccer players (13.2 - 15.1 years) and suggested that maturation influenced technical skills, particularly dribbling skills. In our study only dribbling was "picked-up" in the discriminant analysis, and this likely suggests that the ability to dribble at full speed with changes in direction can best distinguish between selected and non-selected youth Brazilian soccer players.

In soccer, speed, strength and endurance form part of the main abilities of players to express performance during the game (Aquino et al., 2016). In our study selected players significantly out-performed the non-selected ones only in the running speed - $30 \mathrm{~m}$ test, countermovement jump and peak power test. Previous studies with talented players also showed differences in speed and agility (Coelhoe-Silva et al., 2010; Figueiredo et al., 2009), which can be explained by the maturational factor. However, other motor performance markers such as average power, minimum power, the fatigue index, and $\mathrm{VO} 2 \mathrm{max}$, which depend on body size and biological maturation, did not enter the discriminant function. It may be that they were not sensitive enough to discriminate between these players, given that mean differences were of low effect sizes (between 0.23 and 0.39; analysis not shown).

Importantly, the results of our study strongly support the use of objective multivariate profiles in actuarial prediction of selected versus non-selected youth soccer players. As such, they 
can be of great use to coaches, although they are apparently not routinely used ( $\mathrm{O}^{\prime}$ Connor et al., 2016). It is important to note that there are other factors governing the decision to keep or release a player from a club. For example, Huijgen et al. (2014) cite the short term success required in the clubs, which is associated with performance during the games, the number of places available on the team, the coach-athlete as well as directorathlete relationship, playing position, social representation within the team and high performance in a specific dimension.

This study is not without limitations, and at least two of them have to be acknowledged. Firstly, this study was conducted in only one club, with a limited sample size, which limits generalization of the results to other Brazilian clubs. However, previous studies did have similar sample sizes, if not smaller (Archer et al., 2016; Waldron et al., 2014). Secondly, knowledge of the game was analyzed through declarative tactical knowledge and it has limitations. Nevertheless, this test is simple and quick to apply, had the greatest importance in discrimination and has been used and recommended in previous reports (Elferink-Gemser et al., 2010; Huijgen et al., 2014).
This study also has some novel aspects: (1) to the best of our knowledge, this is the first study that discriminates between non-selected and selected elite Brazilian soccer players, (2) a relatively extensive set of variables was considered to define players' multivariate profiles, (3) highly standardized protocols were used, and (4) the results proved to be efficient in supporting coaches' decisions.

In summary, using objective multivariate profiles, this study confirmed club scouts and coaches decisions in their selection processes of U17 soccer players. Declarative tactical knowledge, running speed $-30 \mathrm{~m}$, maturity offset, dribbling, height, and peak power correctly classified $97 \%$ of the selected players. This suggests the usefulness of joint clinical and actuarial prediction in selecting youth soccer players, with high efficiency. Thus, the club scouts and coaches can use, early in the season (i.e., pre-season), objective multivariate profiles as evidence during technical meetings when deciding on players who will be part of the team throughout the season. Adopting this strategy provides a greater chance of success in the selection process of players.

\section{Acknowledgements}

This work was supported by the CNPq and FAPESP [grant number: 2014/16164-5].

\section{References}

Aquino RLQT, Gonçalves LGC, Vieira LH, Oliveira LP, Alves GF, Santiago PRP, Puggina EF. Periodization training focused on technical-tactical ability in young soccer players positively affects biochemical markers and game performance. J Strength Cond Res, 2016; 30: 2723-32

Archer DT, Drysdale K, Bradley E. Differentiating technical skill and motor abilities in selected and nonselected 3-5 year old team-sports players. Hum Mov Sci, 2016; 47: 81-87

Bangsbo J, Iaia FM, Krustrup P. The Yo-Yo intermittent recovery test: a useful tool for evaluation of physical performance in intermittent sports. Sports Med, 2008; 38: 37-51

Blanksby BA, Bloomfield J, Ackland TR, Elliott BC, Morton AR. Athletics, growth and development in children. Camberwell: Harwood Academic Publishers; 1994

Bosco C, Belli A, Astrua M, Tihanyi J, Pozzo R, Kellis S, Tsarpela O, Foti C, Manno R, Tranquilli C. A dynamometer for evaluation of dynamic muscle work. Eur J Appl Physiol, 1995; 70: 379-386

Carling C, Le Gall F, Reilly T, Williams AM. Do anthropometric and fitness characteristics vary according to birth date distribution in elite youth academy soccer players? Scand J Med Sci Sports, 2009; 19: 3-9

Coelho-e-Silva MJ, Figueiredo AJ, Simoes F, Seabra A, Natal A, Vaeyens R, Phillipaerts R, Cumming SP, Malina RM. Discrimination of U-14 Soccer Players by Level and Position. Int J Sports Med, 2010; 31: 790-796

Dawes RM, Faust D, Meehl PE. Clinical versus actuarial judgment. Science, 1989; 243: 1668-1674

Elferink-Gemser MT, Kannekens R, Lyons J, Tromp Y, Visscher C. Knowing what to do and doing it: Differences in self-assessed tactical skills of regional, sub-elite, and elite youth field hockey players. $J$ 
Sports Sci, 2010; 28: 521-528

Figueiredo AJ, Goncalves CE, Coelho e Silva MJ, Malina RM. Characteristics of youth soccer players who drop out, persist or move up. J Sports Sci, 2009; 27: 883-891

Gissis I, Papadopoulos C, Kalapotharakos V, Sotiropoulos A, Komsis G, Manolopoulos E. Strength and speed characteristics of elite, subelite, and recreational young soccer players. Res Sports Med, 2006; 14: 205-214

Giacomini DS, Soares VO, Santos HF, Matias CJ, Greco PJ. Declarative and procedural tactical knowledge in soccer players of different ages. Motricidade, 2011; 7: 43-53

Honer O, Votteler A, Schmid M, Schultz F, Roth K. Psycometric properties of the motor diagnostics in the German football talent identification and development programme. J Sports Sci, 2015; 33: 145-159

Huijgen BCH, Elferink-Gemser MT, Lemmink KAPM, Visscher C. Multidimensional performance characteristics in selected and deselected talented soccer players. Eur J Sport Sci, 2014; 14: 2-10

Huijgen BCH, Elferink-Gemser MT, Post WJ, Visscher C. Soccer skill development in professional. Int J Sports Med, 2009; 30: 585-591

Huijgen BCH, Elferink-Gemser MT, Posto W, Visscher C. Development of dribbling in talented youth soccer players aged 12-19 years: a longitudinal study. J Sports Sci, 2010; 30: 585-591

Jackson AS, Pollock ML, Gettman LR. Intertester reliability of selected skinfold and circumference measurements and percent fat estimates. Res Q, 1978; 49: 546-51

Lago-Penas C, Rey E, Casáis L, Gómez-López M. Relationship between performance characteristics and the selection process in youth soccer player. J Hum Kinet, 2014; 40: 189-199

Lohman TG. Applicability of body composition techniques and constants for children and youths. Exerc Sport Sci Rev, 1986; 14: 325-357

Little T, Williams AG. Specificity of acceleration, maximum speed, and agility in professional soccer players. J Strength Cond Res, 2005; 19, 76-78

Malina RM, Eisenmann JC, Cumming SP, Ribeiro B, Aroso J. Maturity-associated variation in the growth and functional capacities of elite youth football (soccer) players 13-15 years. Eur J Appl Physiol, 2004; 91: 555-62

Malina RM, Cumming SP, Kontos AP, Eisenmann JC, Ribeiro B, Aroso J. Maturity-associated variation in sport-specific skills of youth soccer players aged 13-15 years. J Sports Sci, 2005; 23: 515-22

Matta MO, Figueiredo AJB, Garcia ES, Werneck FZ, Seabra A. Morphological and maturational predictors of technical performance in young soccer players. Motriz, 2014; 20: 280-285

Meylan C, Cronin J, Oliver J, Hughes M. Reviews: Talent identification in soccer: The role of maturity status on physical, physiological and technical characteristics. Int J Sports Sci Coach, 2010; 5: 571-592

Mirwald RL, Baxter-Jones A, Bailey DA, Beunen GP. An assessment of maturity from anthropometric measurements. Med Sci Sports Exerc, 2002; 34: 689-694

O'Connor D, Larkin P, Williams AM. Talent identification and selection in elite youth football: An Australian context. Eur J Sport Sci, 2016; 29: 1-8

Rebelo A, Brito J, Maia J, Coelho-e-Silva MJ, Figueiredo AJ, Bangsbo J, Malina RM, Seabra A. Anthropometric characteristics, physical fitness and technical performance of Under-19 soccer players by competitive level and field position. Int J Sports Med, 2013; 34: 312-317

Reilly T, Williams AM, Nevill A, Franks A. A multidisciplinary approach to talent identification in soccer. J Sports Sci, 2000; 18: 695-702

Siri WE. Body composition from fluid spaces and density: analysis of methods. In: Brozek J, Henschel A. (Eds). Techniques for Measuring Body Composition. Washington: National Academy of Sciences, National Research Council; 1961

Unnithan V, White J, Georgiou A, Iga J, Drust B. Talent identification in youth soccer. J Sports Sci, 2012; 30: 1719-1726

Vaeyens R, Lenoir M, Williams AM, Philippaerts RM. Talent identification and development programmes in sport: Current models and future directions. Sports Med, 2008; 38: 703-714

Vaeyens R, Malina RM, Janssens M, Van Renterghem B, Bourgois J, Vrijens J, Philippaerts RM. A multidisciplinary selection model for youth soccer: the Ghent youth soccer project. Br J Sports Med, 
2006; 40: 928-934

Waldron M, Worsfold PF, Twist C, Lamb K. A three-season comparison of match performances among selected and unselected elite youth rugby league players. J Sports Sci, 2014; 32: 1110-1119

Zacharogiannis E, Paradisis G, Tziortzis S. An evaluation of tests of anaerobic power and capacity. Med Sci Sports Exerc, 2004; 36: 237-242

\section{Corresponding author:}

Rodrigo Aquino, MSc.

Medical School of University of Sao Paulo, Av. Bandeirantes, 3900, 14049-900, Ribeirao Preto, Brazil.

Phone: +55 16 99195-0494

E-mail address: aquino.rlq@gmail.com 